\title{
Efecto in vitro de los extractos hidroalcohólico y etanólico de semilla de papaya (Carica papaya) en Haemonchus contortus
}

\section{In vitro effect of the hydroalcoholic and ethanolic extracts of papaya seeds (Carica papaya) against Haemonchus contortus}

Fecha de recepción: 6 de septiembre de 2017 Fecha de aprobación: 29 de diciembre de 2017
María de la Esperanza Marroquín-Tun ${ }^{1}$

Rosa Isabel Higuera-Piedrahita ${ }^{2}$

María Eugenia López-Arellano ${ }^{3}$

Raquel López-Arellano ${ }^{4}$ Héctor Alejandro de la Cruz-Cruz ${ }^{5}$

Rocío Silva-Mendoza ${ }^{6}$

Jorge Alfredo Cuéllar-Ordaz ${ }^{7}$

DOI: https://doi.org/10.19053/01228420.v15.n1.2018.7756

\section{Resumen}

Entre los principales problemas sanitarios para la producción ovina están los parásitos gastroentéricos; de ellos, el más importante es Haemonchus contortus. Las estrategias actuales de control enfocan la eficacia, junto con el bajo o nulo impacto al ecosistema; el estudio del control integral de parásitos permite la investigación de alternativas con probable efecto antihelmíntico, entre ellas el uso de semilla de papaya (Carica papaya). El objetivo de este trabajo fue evaluar el efecto in vitro de los extractos hidroalcohólico y etanólico de la semilla de papaya (C. papaya) sobre huevos y larvas de $H$. contortus. El trabajo se llevó a cabo con el equipo e instalaciones del Laboratorio 3 de la Unidad de Investigación Multidisciplinaria en la Facultad de Estudios Superiores Cuautitlán, UNAM. Se elaboraron los extractos por medio de macera-

1 Universidad Nacional Autónoma de México (Cuautitlán Izcalli, México).

2 M. Sc. Universidad Nacional Autónoma de México (Cuautitlán Izcalli, México).

3 Ph. D. Centro Nacional de Investigación Disciplinaria en Parasitología Veterinaria (Cuernavaca, Morelos, México).

4 Ph. D. Universidad Nacional Autónoma de México (Cuautitlán Izcalli, México). ORCID: 0000-0002-2050-6119.

5 M. Sc. Universidad Nacional Autónoma de México (Cuautitlán Izcalli, México). delacruz@unam.mx.

6 Universidad Nacional Autónoma de México (Cuautitlán Izcalli, México). jcuellar@unam.mx.

7 M. Sc. Universidad Nacional Autónoma de México (Cuautitlán Izcalli, México). jcuellar@unam.mx. 
ción en solvente. Se inoculó un ovino con 5000 larvas de tercer estadio $\left(\mathrm{L}_{3}\right)$ de $H$. contortus, cepa aislada y mantenida en dichas instalaciones. Se tomaron muestras de materia fecal y se recuperaron huevos para el ensayo de inhibición de la eclosión. Los huevos y las $L_{3}$ fueron pretratados con extracto hidroalcohólico y etanólico de semilla de papaya $(2.5 \mathrm{mg} / \mathrm{ml})$, agua destilada, etanol 90 \% y levamisol $(7.5 \mathrm{mg} /$ $\mathrm{ml}$ ). Se efectuó la técnica de explantes abomasales, evaluando el efecto de los extractos sobre las $\mathrm{L}_{3}$ y su asociación a la mucosa abomasal. Se realizó un ANOVA para muestras repetidas por medio del programa Statgraphics. El extracto etanólico de semilla de papaya mostró el 92 \% de inhibición de la eclosión; el extracto hidroalcohólico mostró $50 \%(p<0.05)$. En el caso de los explantes, el extracto etanólico mostró el $10 \%$ de larvas no asociadas al tejido abomasal, seguido por el extracto hidroalcohólico, con el $60 \%$ de larvas no asociadas $(p<0.05)$. Se concluye que el extracto etanólico tiene efecto sobre huevos, mientras que el extracto hidroalcohólico inhibe la asociación al tejido abomasal.

Palabras clave: Carica papaya; extracto etanólico; extracto hidroalcohólico; Haemonchus contortus.

\begin{abstract}
Among the main health problems for sheep production are gastrointestinal parasites, of which the most important nematode is Haemonchus contortus. Current control strategies focus on the effectiveness, along with the low impact on the ecosystem. The study of the integral control of parasites allows the investigation of alternatives with probable anthelmintic effect, such as the use of papaya (Carica papaya) seeds. This study aimed at evaluating the in vitro effect of hydroalcoholic and ethanolic extracts of papaya seeds on eggs and larvae of $\mathrm{H}$. contortus. The study was carried out with the equipment and facilities of Laboratory 3 of the Multidisciplinary Research Unit at the Facultad de Estudios Superiores Cuautitlán, UNAM. The extracts were prepared through maceration in solvent. A sheep was inoculated with $5000 \mathrm{~L}_{3}$ of $H$. contortus, a strain isolated and maintained in the facilities. Fecal samples were collected, and eggs were recovered for the hatch inhibition assay. The eggs and $L_{3}$ were pretreated with hydroalcoholic and ethanolic extract of papaya seed $(2.5 \mathrm{mg} / \mathrm{ml})$, distilled water, ethanol $90 \%$, and levamisole $(7.5 \mathrm{mg} / \mathrm{ml})$. The abomasal explants technique was used to evaluate the effect of the extracts on $\mathrm{L}_{3}$ and its association to the abomasal mucosa. An ANOVA was performed for repeated samples using the Statgraphics program. The ethanolic extract of the papaya seed inhibited $92 \%$ of hatching, and the hydroalcoholic extract $50 \%(p$ $<0.05)$. In the case of the explants, the ethanolic extract showed $10 \%$ of the larvae not associated with the abomasal tissue, followed by the hydroalcoholic extract with $60 \%$ of non-associated larvae $(p<0.05)$. In conclusion, the ethanolic extract has an effect on eggs, while the hydroalcoholic extract inhibits the association to the abomasal tissue.
\end{abstract}

Keywords: Carica papaya; ethanolic extract; hydro alcoholic extract; Haemonchus contortus; seeds.

\title{
Para citar este artículo:
}

Marroquín-Tun MA., Higuera-Piedrahita RI., López-Arellano ME., López-Arellano R., Cruz-Cruz HA., Silva-Mendoza R., Cuéllar-Ordaz JA. Efecto in vitro de los extractos hidroalcohólico y etanólico de semilla de papaya (Carica papaya) en Haemonchus contortus. Ciencia y Agricultura. 2018; 15(1): 53-59. 


\section{Introducción}

El estatus sanitario de un rebaño ovino garantiza la producción y la generación de indicadores positivos; unas de las enfermedades que afectan este rubro son las parasitosis gastroentéricas (1), de las cuales sobresale, por su característica hematófaga, la ocasionada por el nematodo Haemonchus contortus (2). El manejo sanitario actual impide el control de infecciones y maximiza el daño causado en el hospedero, además de originar otro tipo de problemas, como la resistencia antiparasitaria; en estas condiciones, herramientas alternativas, como la herbolaria y la homeopatía, y el uso de razas resistentes abren paso a la investigación $(3,4)$.

El control integrado de parásitos plantea una herramienta tangible a los problemas suscitados por el manejo señalado; dentro de las opciones se encuentra la herbolaria, que permite el uso de diferentes plantas en preparaciones hidroalcohólicas, alcohólicas o acuosas que potencian el efecto de las moléculas activas (5-9). La semilla de papaya se ha utilizado como principio antihelmíntico (10) en extractos acuosos, pero su eficacia es controvertida; el solvente que se utiliza para la extracción y los resultados reportados son diversos (11). La semilla de papaya debe tener un manejo previo, como el secado, oreado, macerado y mezcla con el solvente final (12). El objetivo de este trabajo fue evaluar el efecto del extracto hidroalcohólico y del etanólico de la semilla de papaya (Carica papaya) contra huevos y $L_{3}$ del parásito $H$. contortus.

\section{Materiales y métodos}

\section{A. Localización}

Este trabajo se llevó a cabo en el Laboratorio 3 de la Unidad de Investigación Multidisciplinaria, de la Facultad de Estudios Superiores Cuautitlán, UNAM. La localidad está a 2,100 m s.n.m., posee clima semiseco y una temperatura media anual de $16.6{ }^{\circ} \mathrm{C}(23)$.

\section{B. Extracto etanólico e hidroalcohólico de semilla de papaya (C. papaya)}

La metodología utilizada es la reportada por Bautista (13). Se obtuvieron $400 \mathrm{~g}$ de semillas de papaya (C. papaya) recién cortada, adquirida en el mercado regional; a las semillas se les extrajo la humedad en estufa a $100{ }^{\circ} \mathrm{C}$ durante 48 horas, obteniéndose $68 \mathrm{~g}$ en base seca.

Se realizó el macerado de los 68 g de semillas (base seca) en mortero; $34 \mathrm{~g}$ se mezclaron con solvente hidroalcohólico (50:50) y $34 \mathrm{~g}$ se destinaron para el extracto etanólico al $90 \%$. Durante cinco días se adicionó al macerado, de forma lenta, solvente a $15{ }^{\circ} \mathrm{C}$, hasta que la semilla perdió su color. El extracto obtenido se concentró por medio de rotaevaporador y el residuo obtenido se liofilizó.

\section{Larvas de $\boldsymbol{H}$. contortus}

Los huevos de $H$. contortus se obtuvieron de las heces de un cordero donador, infectado experimentalmente con 5000 larvas de tercer estadio $\left(\mathrm{L}_{3}\right)$. La cepa es de origen ovino aislada, mantenida y caracterizada en la Facultad de Estudios Superiores Cuautitlán (14). Se colectaron larvas infectantes por medio de la técnica de Corticelli-Lai (15).

1) Técnica de inhibición de la eclosión de huevos. Esta técnica se realizó siguiendo la metodología citada por Coles y col. (16) y Carvalho y col. (17).

1. Se homogeneizaron las muestras de materia fecal con un agitador en solución salina fisiológica.

2. De las dos fases formadas, se colectó el sobrenadante por pipeteo en un tubo para centrifugar.

3. Se procedió a centrifugar a $3000 \mathrm{~g} / 5 \mathrm{~min}$, hasta que los huevos estuvieron en el fondo de los tubos, realizando siete repeticiones de este paso.

4. Una vez que se obtuvieron los huevos se procedió a cuantificarlos. Se aplicaron los siguientes tratamientos como se muestra en la Tabla I. 
Tabla I. Tratamientos aplicados a huevos de $H$. contortus.

\begin{tabular}{lc}
\hline \multicolumn{1}{c}{ Tratamiento } & Dosis \\
\hline Albendazol & $5 \mathrm{mg} / \mathrm{ml}$ \\
Extracto hidroalcohólico & $2.5 \mathrm{mg} / \mathrm{ml}$ \\
Extracto etanólico & $2.5 \mathrm{mg} / \mathrm{ml}$ \\
Agua destilada & $1 \mathrm{ml}$ \\
Etanol & $1 \mathrm{ml}$ \\
\hline
\end{tabular}

5. Se adicionaron aproximadamente 100 huevos a cada pozo.

6. Se llevó a incubación a $27^{\circ} \mathrm{C}$ durante 48 horas.

7. Se llevó a cabo la lectura al microscopio con el objetivo de 40X, se añadieron gotas de Lugol para fijar la muestra y se cuantificó.

2) Técnica de explantes abomasales. Se realizó según la técnica descrita por Jackson y col. (18). El abomaso se obtuvo de un ovino libre de nematodos gastroentéricos. Para obtener los explantes se incidió por la curvatura menor, se retiró el contenido y se lavó con solución salina fisiológica al $0.85 \%$, se realizaron cortes circulares de tres centímetros de diámetro y se colocaron en placas de cultivo con medio Hanks y Hepes a $20 \mathrm{mmol}$ y 37 ${ }^{\circ} \mathrm{C}$.

A las $L_{3}$ obtenidas se les realizó el siguiente proceso:

1. Se desenvainaron con hipoclorito de sodio al $1 \%$ y se centrifugaron a $3500 \mathrm{~g} / 5 \mathrm{~min}$.

2. Se realizaron tres lavados con agua destilada y se centrifugaron a $3500 \mathrm{~g} / 5 \mathrm{~min}$.

3. Se aplicaron los siguientes tratamientos, como se muestra en la Tabla II.
Tabla II. Tratamientos aplicados a las $\mathrm{L}_{3}$ de $H$. contortus desenvainadas.

\begin{tabular}{ll}
\hline \multicolumn{1}{c}{ Tratamiento } & \multicolumn{1}{c}{ Dosis } \\
\hline Levamisol & $7.5 \mathrm{mg} / \mathrm{ml}$ \\
Extracto hidroalcohólico & $2.5 \mathrm{mg} / \mathrm{ml}$ \\
Extracto etanólico & $2.5 \mathrm{mg} / \mathrm{ml}$ \\
Agua destilada & $1 \mathrm{ml}$ \\
Etanol & $1 \mathrm{ml}$ \\
\hline
\end{tabular}

4. Se adicionaron $2500 \pm 200 \mathrm{~L}_{3}$ de $H$. contortus a cada pozo sobre el tejido abomasal.

5. Se incubaron durante tres horas y se realizaron dos lavados del tejido con solución salina fisiológica a $0.85 \%$ a $37^{\circ} \mathrm{C}$.

6. El tejido abomasal se sometió a digestión con una mezcla de ácido clorhídrico al $1 \%$ y pepsina al $1 \%$ durante 48 horas.

7. Una vez transcurrido este tiempo se procedió a cuantificar las $\mathrm{L}_{3}$ asociadas al tejido.

\section{Análisis estadístico}

Los datos fueron ajustados a una curva de distribución normal por medio del programa Statgraphics $\mathrm{XV}^{\circledR}$, y posteriormente se realizó un ANOVA multifactorial por medio del mismo programa.

\section{Nota ética}

El manejo del cordero donador de huevos de $H$. contortus se realizó conforme a los lineamientos del Comité Institucional para el Cuidado y Uso de los Animales de Experimentación, de la Facultad de Estudios Superiores Cuautitlán de la Universidad Nacional Autónoma de México (CICUAEFESC-UNAM). 


\section{Resultados y discusión}

\section{A. Técnica de inhibición de la eclosión de huevos}

El extracto hidroalcohólico mostró inhibición de la eclosión de huevos del 50 \%; el extracto etanólico, del $90 \%$; el albendazol, del $78 \%$, mientras que el testigo con agua mostró $100 \%$ de desarrollo a $\mathrm{L}_{1}$, los resultados se detallan en la Figura 1.

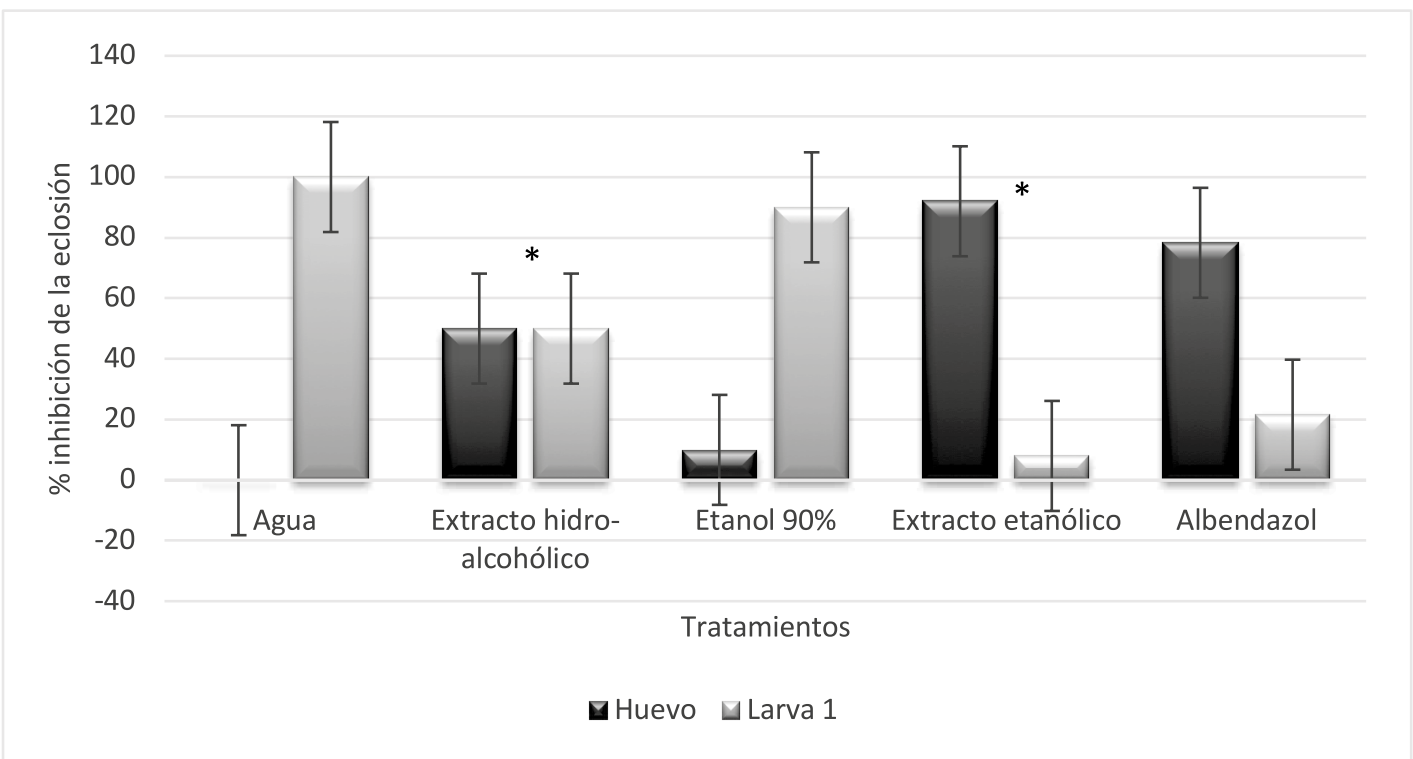

Fig. 1. Inhibición de la eclosión de huevos de $H$. contortus expuestos a extracto etanólico y extracto hidroalcohólico de semilla de papaya (C. papaya). * Muestran diferencia estadísticamente significativa $(p<0.05) \mathrm{ME} \pm \mathrm{EE}$.

Batatinha y col. (19) mostraron que la actividad antihelmíntica atribuida a la semilla de papaya se debe al compuesto químico denominado benzil isotiocianato. Kermanshai y col. (20) mostraron una correlación positiva entre la actividad antihelmíntica y la presencia de benzil isotiocianato en los aceites esenciales de las semillas de papaya. Batatinha y col. (19) mostraron reducción del metabolismo energético e interferencia motora de Ascaridia galli, por lo cual presumieron parte del mecanismo de acción. Los resultados descritos son comparables con los obtenidos en el presente estudio, donde el extracto etanólico de la semilla de papaya mostró inhibición de la eclosión de huevos de $\mathrm{H}$. contortus. De la misma manera, Buttle y col. (22) mostraron que el mecanismo antihelmíntico de la semilla de papaya se debe a cistein proteinasas, el cual se demostró en ensayos in vivo e in vitro en roedores. Las enzimas presentes en la semilla de papaya se unen a proteínas presentes en la cutícula del parásito adulto, causando debili- tamiento, ruptura y liberación de tejidos internos, con consecuente muerte del adulto. Estos hallazgos explican la ruptura de la cutícula del huevo, causando liberación y muerte de este (13).

\section{B. Técnica de explantes abomasales}

El extracto etanólico de semilla de papaya (C. papaya) mostró un $10 \%$ de larvas no asociadas al tejido abomasal; en el caso del extracto hidroalcohólico, el $60 \%$ de las larvas aplicadas no se asociaron al tejido abomasal; el levamisol evidenció $0 \%$ de larvas asociadas al tejido, mientras que el etanol mostró un $79 \%$ de larvas asociadas al tejido abomasal; los resultados se muestran en la Figura 2 . 


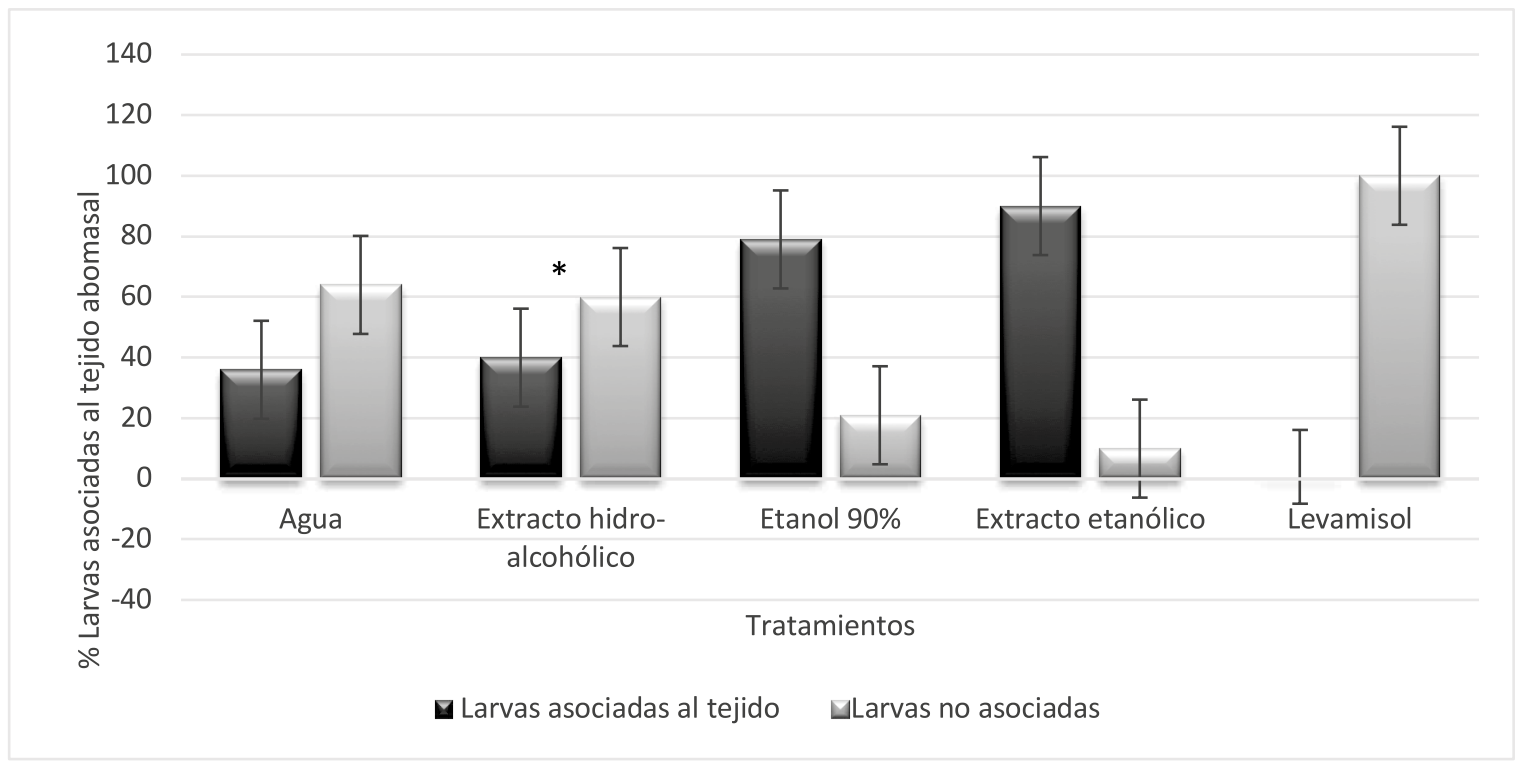

Fig. 2. Evaluación de larvas asociadas y no asociadas al tejido abomasal, las $L_{3}$ de $H$. contortus fueron expuestas a extracto etanólico y extracto hidroalcohólico de semilla de papaya (C. papaya). * Muestran diferencia estadísticamente significativa $(p<0.05) \mathrm{ME} \pm \mathrm{EE}$.

Batatinha y col. (19) mostraron que extractos acuosos hechos con semilla de papaya (C. papaya) muestran actividad antihelmíntica, resultado relacionado con los encontrados en este estudio, donde el $60 \%$ de las larvas no se asociaron al tejido abomasal en el ensayo de explantes abomasales. Kermanshai y col. (20) mostraron que el compuesto responsable (benzilisotiocianato) es soluble en agua, lo que podría ser responsable de la actividad de los extractos acuosos. Sin embargo, estudios hechos con extractos acuosos de semilla de papaya mostraron un $18.6 \%$ de eficacia sobre nematodos adultos (13).

\section{Conclusiones}

El extracto etanólico de semilla de papaya (C. papaya) tiene efecto sobre huevos, inhibiendo su eclosión en un $92 \%$; en la técnica de explantes abomasales el extracto hidroalcohólico mostró efecto del $60 \%$, impidiendo la asociación de $\mathrm{L}_{3}$ al tejido abomasal.

\section{Agradecimientos}

Al proyecto PAPIIT/UNAM 28IN226217, Efecto antihelmíntico del extracto etanólico de Artemisia cina, semilla de papaya (Carica papaya) y taninos condensados sobre el nematodo hematófago Haemonchus contortus, por el financiamiento de este trabajo.

A la unidad de helmintos del Instituto Nacional de Investigaciones Forestales, Agrícolas y Pecuarias (INIFAP), CENID-PAVET.

AI CONACyT, por el apoyo económico al becario.

\section{Referencias}

(1) Hotez PJ., Brindley PJ., Bethony JM., King CH., Pearce EJ., Jacobson J. Helminth infections: the great neglected tropical diseases. J. of Clin. Inv. 2008; 118: 1311-1321. DOI: http://doi. org/10.1172/JCl34261.

(2) Busin V., Sargison ND. Preliminary observations on the value of using effective anthelmintic drugs to control nematode parasitism in lambs in the face of a high level of infective larval challenge. Small Rum. Res. 2014; 119: 172-175. DOI: http://doi. org/10.1016/j.smallrumres.2014.02.001.

(3) Muñiz Lagunes A., González Garduño R., López Arellano ME., Ramírez Valverde R., Ruíz Flores A., et al. Anthelmintic resistance in gastrointestinal nematodes from grazing beef cattle in Campeche State, México. Trop. Anim Health Prod. 2015; 47(6): 1049-1054. DOI: http://doi.org/10.1007/s11250-015-0826-3.

(4) Waller PJ. From discovery to development: current industry perspectives for the development of novel methods of helminth con- 
trol in livestock. Vet Parasitol. 2006; 139: 1-14. DOI: http://doi. org/10.1016/j.vetpar.2006.02.036.

(5) Klimpel S., Abdel Ghaffar F., Al Rasheid KA., Aksu G., Fischer K., Strassen B., Mehlhorn H. The effects of different plant extracts on nematodes. Parasitol Res. 2011; 108(4): 1047-1054. DOI: http:// doi.org/10.1007/s00436-010-2168-4.

(6) Knox M., Torres Acosta JFJ., Aguilar Caballero A. Exploiting the effect of dietary supplementation of small ruminants on resilience and resistance against gastrointestinal nematodes. Vet. Parasitol. 2006; 139: 385-393. DOI: http://doi.org/10.1016/j. vetpar.2006.04.026.

(7) Martínez Ortiz C., Vargas Magaña JJ., Aguilar Caballero AJ., Sandoval Castro CA., Cob Galera L., May Martínez M., et al. Combining the effects of supplementary feeding and copper oxide needles improves the control of gastrointestinal nematodes in browsing goats. Vet. Parasitol. 2007; 146: 66-76. DOI: http://doi. org/10.1016/j.vetpar.2007.02.012.

(8) Torres Acosta JFJ., Hoste H. Alternative or improved methods to limit gastro-intestinal parasitism in grazing sheep and goats. Small Rum. Res. 2008; 77: 159-173. DOI: http://doi.org/10.1016/j. smallrumres.2008.03.009.

(9) Rocha RA., Bresciani KDS., Barros TFM., Fernandes LH., Silva MB., Amarante AFT. Sheep and cattle grazing alternately: nematode parasitism and pasture decontamination. Small Rum. Res. 2008; 75: 135-143. DOI: http://doi.org/10.1016/j.smallrumres.2007.09.001.

(10) Omayma M., Abou S., Maha M., Olfat A., Nardden Zakka, B. The effect of praziquantel and Carica papaya seeds on Hymenolepis nana infection in mice using scanning electron microscope. Parasitol Res. 2014; 113: 2827- 2836. DOI: http://doi. org/10.1007/s00436-014-3943-4.

(11) Molento MB. Parasite control in the age of drug resistance and changing agricultural practices. Vet. Parasitol. 2009; 163: 229234. http://doi.org/10.1007/s00436-014-3943-4.

(12) Bar Nun N., Mayer AM. Cucurbitacins protect cucumber tissue against infection by Botrytis cinerea. Phytochemistry. 1990; 29:787-791. DOI: http://doi.org/10.1016/0031-9422(90)80019-D.

(13) Bautista O., Ovalle C. Evaluación de la eficacia de un preparado acuoso de semilla de papaya (Carica papaya) contra Haemonchus contortus en ovinos con infección artificial. Tesis de licenciatura. Universidad Nacional Autónoma de México. Facultad de Estudios Superiores Cuautitlán. 2012.

(14) Valdez Ramírez L., Cuéllar Ordaz J. Aislamiento de una cepa de Haemonchus contortus de origen ovino. Tesis que para obtener el título de Médico Veterinario Zootecnista. Facultad de Estudios Superiores Cuautitlán- UNAM. 2009.

(15) Van Wyk J., Mayhew E. Morphological identification of parasitic nematode infective larvae of small ruminants and cattle: A practical lab guide. J. Vet. Res. 2013; 80: 1-14. DOI: http://doi. org/10.4102/ojvr.v80i1.539.

(16) Coles G., Borgsteede F., Geerts S., Klei T., Taylor M., Waller P. World Association for the Advancement of Veterinary Parasitology (W.A.A.V.P.) methods for the detection of anthel- mintic resistance in nematodes of veterinary importance. Vet. parasitol. 1992; 44: 35-44. DOI: http://doi.org/10.1016/03044017(92)90141-U.

(17) Carvalho C., Chagas A., Cotinguiba F., Furlan M., Brito L., Chaves C., et al. The anthelmintic effect of plant extracts on Haemonchus contortus and Strongyloides venezuelensis. Vet. Parasitol. 2012; 183: 260-268. DOI: http://doi.org/10.1016/j. vetpar.2011.07.051.

(18) Jackson F., Greer A., Huntley J., McAnulty R., Bartley D., Stanley A., et al. Studies using Teladorsagia circumcincta in an in vitro direct challenge method using abomasal tissue explants. Vet. Parasitol. 2004; 124: 73-89. DOI: http://doi.org/10.1016/j. vetpar.2004.06.025.

(19) Batatinha M., Santos M., Botura M., Almeida G., Domingues J., Almeida M. Efeitos in vitro dos extratos de folhas de Musa cavendishii Linn e de sementes de Carica papaya Linn sobre culturas de larvas de nematódeos gastrintestinais de caprinos. Rev. Bras. PL. Med. Botucatu. 2004; 7(1): 11-15.

(20) Kermanshai R., McCarry BE., Rosenfeld J. Benzylisothiocyanate is the chief or sole anthelmintic in papaya seed extracts. Phytochemistry. 2001; 57: 427-435. DOI: http://doi.org/10.1016/ S0031-9422(01)00077-2.

(21) Kumar D., Mishra S., Tripathi H. Mechanism of anthelmintic action of benzylisothiocyanate. Fitoterapia. 1991; 65(5): 403-410.

(22) Buttle D., Behnke J., Bartley Y., Elsheikha H., Bartley D., Garnett M. Oral dosing with papaya latex is an effective anthelmintic treatment for sheep infected with Haemonchus contortus. Parasites and vectors. 2001; 4-36.

(23) Reyna T. Características climático-frutícolas en Cuautitlán Estado de México. Invest. Geog. 1977; 8: 55-66. 
